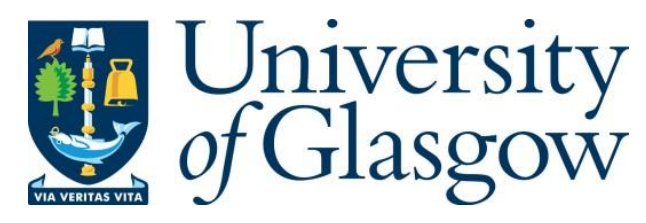

Patel, J., Fioranelli, F., Ritchie, M. and Griffiths, H. (2019) Fusion of deep representations in multistatic radar networks to counteract the presence of synthetic jamming. IEEE Sensors Journal, 19(15), pp. 6362-6370.

(doi:10.1109/JSEN.2019.2909685)

There may be differences between this version and the published version. You are advised to consult the publisher's version if you wish to cite from it.

http://eprints.gla.ac.uk/183430/

Deposited on: 3 April 2019

Enlighten - Research publications by members of the University of Glasgow http://eprints.gla.ac.uk 


\title{
Fusion of Deep Representations in Multistatic Radar Networks to Counteract the Presence of Synthetic Jamming
}

\author{
Jarez S. Patel, Student Member, IEEE, Francesco Fioranelli, Matthew Ritchie, \\ Member, IEEE and Hugh Griffiths, Fellow, IEEE
}

\begin{abstract}
Micro-Doppler signatures are extremely valuable in the classification of a wide range of targets. This work investigates the effects of jamming on micro-Doppler classification performance and explores a potential deep topology enabling low bandwidth data fusion between nodes in a multistatic radar network. The topology is based on an array of three independent deep neural networks (DNNs) functioning cooperatively to achieve joint classification. In addition to this, a further DNN is trained to detect the presence of jamming and from this it attempts to remedy the degradation effects in the data fusion process. This is applied to real experimental data gathered with the multistatic radar system NetRAD, of a human operating with seven combinations of holding a rifle-like object and a heavy backpack which is slung on their shoulders. The resilience of the proposed network is tested by applying synthetic jamming signals into specific radar nodes and observing the networks' ability to respond to these undesired effects. The results of this are compared with a traditional voting system topology, serving as a convenient baseline for this work.
\end{abstract}

Index Terms - Radar; Multistatic Radar; Human Micro Doppler; Radar Classification; Fusion; DNN; Synthetic Jamming

\section{INTRODUCTION}

$\mathrm{T}$ he Doppler effect is a directly observable phenomenon in radar and is detected through the change in received frequency as a target approaches or recedes from the transmitter. Micro-Doppler is exhibited through additional modulations on top of the main Doppler component. This effect is heavily utilized in Automatic Target Recognition (ATR) applications and classification problems, as these further modulations are often unique to a target [1]-[4]. From this it is possible to discriminate between a variety of human activities such as walking, running, crawling and the

Manuscript received DATE; revised DATE; accepted DATE. Date of publication DATE; date of current version DATE. The associate editor coordinating the review of this paper and approving it for publication was REVIEWER. (Corresponding author: J. S. Patel).

J. S. Patel and F Fioranelli are with the School of Engineering, University of Glasgow, G12 8QQ, Glasgow UK

M. Ritchie and H. Griffiths are with the Electronic \& Electrical Engineering, University College London, WC1E 7JE, London UK (e-mail jarez.patel@glasgow.ac.uk ; $\quad$ francesco.fioranelli@glasgow.ac.uk; m.ritchie@ucl.ac.uk h.griffiths@ucl.ac.uk ) Digital Object Identifier DOI NUM characterization of free or confined movements of the arms, related to the carrying of different objects this may potentially include weapons such as rifles [5].

Machine learning (ML) has become incredibly popular over the past few years and it has found many applications in engineering and data science fields [6], as well as making a significant impact in radar research. This is owed to the simple fact that ML provides an opportunistic means of processing or analyzing a given data set, which requires a degree of intelligence which can conveniently be tackled through nonlinear extrapolation. Typically, in classification problems, features are extracted through using a bespoke algorithm which is generally tailored to the mission at hand, this can be laborious and may be considered inadequate in terms of system robustness (depending on the application). Specifically, in the context of classification in micro-Doppler the spectrogram can vary drastically depending on the aspect angle [7][8], radar parameters and many other operating conditions. Therefore, designing a robust feature extraction algorithm which can cope under this challenging environment is incredibly difficult. It is also clear that the classification accuracy is highly dependent upon the precision at which the algorithm can extract the most important features [9][10].

Deep learning is derived from the same fundamental concepts which ML is based upon, specifically neural networks $(\mathrm{NN})$, however it differs in that it consists of multiple processing layers of diverse dimensions, each designed to perform a certain task within the context of the network optimally. An extensive variety of structures and topologies have been rigorously researched, to name but a few examples: Convolutional Neural Networks (CNN), Long Short Term Memory (LSTM), Deep Belief Network (DBN) and DNN [11]-[16]; these enable high classification accuracies to be obtained over large data sets of images.

Multistatic radar offers many advantages over traditional monostatic radar, the primary one being, that it enables a significantly increased coverage and provides a multiperspective view of the target [17]. Such configurations also boast resilience to jamming, though necessary systems need to be in place to actively detect and cooperatively manage corrupted sensor information. A recent publication investigated classification performance with the same multistatic radar with degraded radar data as a function of the manipulated number of pulses [18]. This utilized a feature extraction algorithm which 
exploited eight empirical and statistical features to perform classification; and as expected, accuracy is hindered as the severity of degradation is increased.

Deep learning has not been leveraged or copiously applied in the context of multistatic radar alongside the presence of targeted enemy jamming. The aim of this work is to exploit the inherent Micro-Doppler effect in conjunction with deep learning algorithms, to enable a cooperative ATR approach to a human target performing seven, potentially hostile activities in an open field. Multi-domain frequency representations of the target will also be exploited to see which transformation is the most beneficial to the classification task [19], implementing multiple of these to improve the overall performance of the system. Intelligent data fusion amongst the radar nodes and active jamming detection systems and topologies will also be investigated; to observe how the multistatic radar system can cope under the presence of an array of mostly hostile jamming measures, simulating purposeful enemy interference.

The rest of this paper is organized as follows: Section II presents the experimental setup and the parameters used in the radar system, Section III details the signal processing methods utilized, Section IV presents the configuration of the networks, Section V presents results and discusses noteworthy findings, finally, Section VI concludes the work and Section VII indicates areas for future research.

\section{EXPERIMENTAL SETUP \& RADAR SYSTEM}

The multistatic radar system (NetRAD) which has been developed over the past 15 years at University College London (UCL) was used to collect the experimental data presented in this paper. It is a coherent pulsed radar with three separate but identical nodes operating at S-Band [20]. The transmitted waveform is a linear frequency modulated (LFM) chirp signal, which enables stretch processing and pulse compression at the receiver [17]. The operating parameters surrounding the radar system are detailed in Table. I; the antennas used for these experiments were parabolic dish antennas which have a beamwidth of approximately $18^{\circ} \times 18^{\circ}$, yielding a cross range resolution of approximately $28 \mathrm{~m}$ at a range of $90 \mathrm{~m}$. This allowed the area in which the target is operating within to be well encapsulated. The bandwidth chosen led to a range resolution of $3.3 \mathrm{~m}$ and combining this with the pulse length leads to a pulse compression ratio of 27 or an equivalent gain of $14.3 \mathrm{~dB}$.

TABLE. I

OPERATIONAL PARAMETERS OF THE MULTISTATIC RADAR SYSTEM (NETRAD)

\begin{tabular}{cc}
\hline \hline Radar Parameter & Value \\
\hline Center Frequency $\left(F_{c}\right)$ & $2.4 \mathrm{GHz}$ \\
Transmit Power $\left(P_{t}\right)$ & $+23 \mathrm{dBm}$ \\
Bandwidth $\left(B_{W}\right)$ & $45 \mathrm{MHz}$ \\
Pulse Repetition Frequency $(P R F)$ & $5 \mathrm{KHz}$ \\
Antenna Gain $\left(G_{T X}, G_{R X}\right)$ & $18 \mathrm{dBi}$ \\
Pulse Length $(\tau)$ & $0.6 \mu \mathrm{s}$ \\
Recording Time & $10.0 \mathrm{~s}$ \\
\hline \hline
\end{tabular}

The experiments were performed in a large open field at the UCL sports ground in Shenley during March 2016. The setup chosen deployed three radar nodes along a linear baseline with $50 \mathrm{~m}$ separation, as depicted in Fig. 1a. The target operated at an average distance of $90 \mathrm{~m}$ from the transmitter (Node 1) and $103 \mathrm{~m}$ from the bistatic receiver (Node 3). Node 1 operated as a monostatic transceiver in VV-pol, Node 2 was configured as a monostatic receiver in horizontal polarization (H-Pol), therefore functioning in $\mathrm{VH}-\mathrm{Pol}$ and Node 3 was configured as a bistatic receiver in VV-pol. The target carried a metallic pole (in relevant tests) of $1 \mathrm{~m}$ in length, to mimic the constrained movement of holding a rifle (Fig. 1b) and a heavy rucksack weighing approximately $15 \mathrm{~kg}$, as shown in Fig. 1c.



(a)

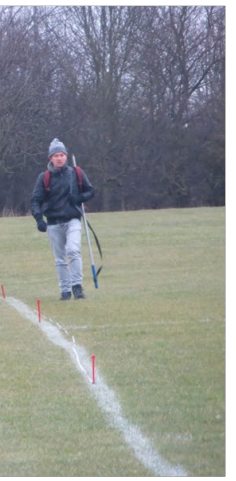

(b)



(c)
Fig. 1. Experimental configuration and an example of a subject performing some activities down range

The data collection consisted of each person (four people) performing seven activities of combinations of walking with a rucksack and with a rifle. These activities and the corresponding class identifiers are detailed in Table. II.

TABLE. II

DESCRIPTION OF HUMAN ACTIVITIES/CLASSES

\begin{tabular}{ccc}
\hline \hline $\begin{array}{c}\text { Class } \\
\text { Identifier } /\end{array}$ & \multicolumn{2}{c}{ Description of physical activity } \\
\cline { 2 - 3 } $\boldsymbol{\zeta}$ & Rucksack & Rifle \\
\hline $\mathbf{0}$ & N/A & N/A \\
$\mathbf{1}$ & Strap on one shoulder & N/A \\
$\mathbf{2}$ & Strap on both shoulders & N/A \\
$\mathbf{3}$ & Slung on one shoulder & Held with two hands across \\
$\mathbf{4}$ & N/A & Held with two hands across \\
$\mathbf{5}$ & Strap on both shoulders & Slung on one shoulder \\
$\mathbf{6}$ & Strap on both shoulders & Held with two hands across \\
\hline \hline
\end{tabular}

${ }^{\dagger}$ The subject walks forward (towards node 1) in all the configurations.

\section{Signal PROCESSING}

\section{A. Radar Data Processing}

A comprehensive dataset was gathered consisting of 10 repeats per person ( 40 samples) of the 7 different activities (280) over the three radar nodes (840 total samples). The recording duration of each sample was 10 seconds, each of these were matched filtered by performing a Hilbert 
transformation and correlating against a bank of reference chirp signals. Once the range time plot was produced a Constant False Alarm Rate (CFAR) detector algorithm was applied to automatically detect the range cells in which the human targets operated within, which differed between the nodes and occasionally samples. The number of training cells used was 20, with 2 guard cells and a probability of false alarm (PFA) of 0.01. This also ensured that the subsequent processing stages were as clean as possible, as empty range cells were not dwelled upon.

A Short Time Fourier Transform (STFT) was then applied over these identified range cells, with a pad factor of 4 , a hamming window of length $0.3 \mathrm{~s}$ and a $95 \%$ overlap factor [5] [7]. These samples were then split into $10(1.0 \mathrm{~s}$ each $)$ yielding a total of 8400 spectrogram samples across the whole system. To provide the deep network with a further representation of the input samples, a Cadence Velocity Diagram (CVD) was produced by taking the FFT across each successive Doppler bin [21]. An example of some spectrograms obtained for two different classes and the corresponding CVD is shown in Fig. 2. The cepstrogram transformation which reveals logfrequency change over time, was investigated but the plots obtained were found to be unfavorable for classification [24].

The entire signal processing chain was fully automated without the need of manual inputs once the initial processing parameters had been set and could be executed through a single command. If the script had control over the radar parameters, it could be dropped into a closed loop radar system with very little modification.
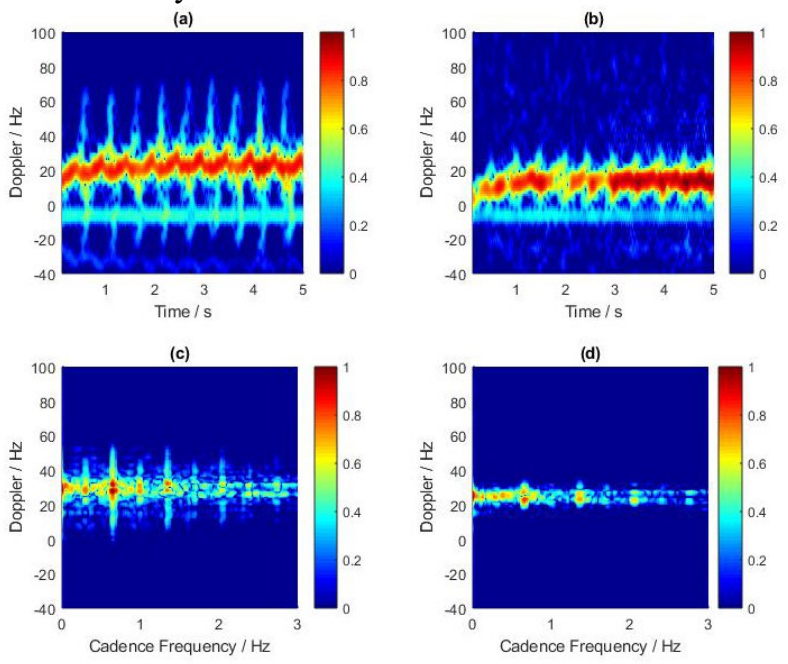

Fig. 2. Micro-Doppler signatures as a spectrogram and CVD (normalised) : (a) Normal walking spectrogram / $\zeta_{0}$; (b) Walking with rucksack spectrogram / $\zeta_{2} ;$ (c) Normal walking CVD / $\zeta_{0} ;$ (d) Walking with rucksack CVD / $\zeta_{2}$.

\section{B. Synthetic Jamming Injection}

To simulate potential scenarios of jamming, a methodology was devised whereby commonly used and well understood electronic attack (EA) methods were borrowed from the field of electronic warfare (EW) [22] and adapted to suit the experimental setup. A summary of the implemented techniques and their corresponding class identifiers are detailed in Table. III.

TABLE. III

COMBINATIONS OF JAMMING TYPES, TECHNIQUES AND A DESCRIPTION OF THE METHOD OF IMPLEMENTATION BOTH VIRTUALLY AND PHYSICALLY

Jamming

Type Technique

\begin{tabular}{|c|c|c|}
\hline Escort & $\begin{array}{l}\text { Targeted } \\
\text { Pulse } \\
\text { Compression } \\
/ \varsigma_{1}-\varsigma_{3}\end{array}$ & $\begin{array}{l}\text { High level of jamming whereby the transmitted } \\
\text { waveform is learnt and it retransmits modifying } \\
\text { or removing or the initial frequency modulation } \\
\text { - a dedicated vehicle retrofitted for electronic } \\
\text { attack }\end{array}$ \\
\hline $\begin{array}{l}\text { Self- } \\
\text { Protect } \\
\text {-ion }\end{array}$ & $\begin{array}{l}\text { Spot } \\
/ \boldsymbol{\varsigma}_{4}-\boldsymbol{\varsigma}_{6}\end{array}$ & $\begin{array}{l}\text { Random noise injected throughout the range } \\
\text { cells that the target is operating within - a simple } \\
\text { dirty handheld jammer }\end{array}$ \\
\hline \multirow[b]{4}{*}{$\begin{array}{l}\text { Stand } \\
\text { in }\end{array}$} & $\begin{array}{l}\text { Cover } \\
/ \varsigma_{7}-\varsigma_{9}\end{array}$ & $\begin{array}{l}\text { Noise superimposed onto random frequency } \\
\text { modulations - dedicated kit which another } \\
\text { soldier could carry }\end{array}$ \\
\hline & $\begin{array}{l}\text { Blinking } \\
/ \varsigma_{10}-\varsigma_{12}\end{array}$ & $\begin{array}{l}\text { Similar to spot jamming, however jamming is } \\
\text { alternated at } 2 \mathrm{~Hz} \text {, in this scenario there is no } \\
\text { second target in the field - dedicated kit to } \\
\text { provide coordinated jamming }\end{array}$ \\
\hline & $\begin{array}{l}\text { Unintentional } \\
\text { /Inadvertent } \\
\text { / } \varsigma_{13}-\varsigma_{15}\end{array}$ & $\begin{array}{l}\text { Low level of jamming, whereby random bursts } \\
\text { of noise are observed extending across the entire } \\
\text { processing range of radar - communications } \\
\text { within the area of detection are inadvertently in } \\
\text { operation during the time of recording (such as } \\
\text { Wi-Fi) }\end{array}$ \\
\hline & $\begin{array}{c}\text { Deceptive/ } \\
\varsigma_{16}-\varsigma_{18}\end{array}$ & $\begin{array}{l}\text { Very high level of jamming, with similar } \\
\text { methodology to targeted pulse compression, } \\
\text { however the position of the target is constantly } \\
\text { shuffled between range bin extents on a pulse to } \\
\text { pulse basis. Consequently, the CFAR detector } \\
\text { output becomes erratic - an advanced device } \\
\text { inconspicuously deployed close to the radar, } \\
\text { providing intelligent directed jamming into that } \\
\text { node }\end{array}$ \\
\hline
\end{tabular}

In each of the listed techniques, three levels of jamming severity were employed; this is discussed in detail in Section V.C. There are 6 jamming implementations, 3 levels (18) and a further class ( $\left.\varsigma_{0}\right)$ to represent an uncorrupted case (19) and two transformations types, leading to a total of 38 jamming classes. The jamming to signal ratio (JSR) was determined for each step and was evaluated based on Eqn. 1, this ensures that the values are feasible for a realistic jammer; the equation below represents a self-protection jammer (SPJ).

$$
J S R=\frac{\overline{P_{J}} G_{J}^{2} B_{W}^{R} J_{e f f}}{\tau P_{p k}^{R} f_{P R F} G_{p c} G_{R}^{2} B_{W}^{J}} \cdot \frac{4 \pi R^{2}}{\sigma}
$$

The terms in Eqn. 1 are: $P_{J}$ represents the average transmit power, $G_{J}$ the antenna gain and $B_{W}^{J}$ the bandwidth, collectively for the jamming device. $B_{W}^{R}$ is the bandwidth, $\tau$ is pulse length, $p_{p k}^{R}$ is the peak transmit power, $f_{P R F}$ is the PRF, $G_{p c}$ is the pulse compression gain, $G_{R}$ is the antenna gain, $R$, is range and $\sigma$ is the radar cross section (RCS), all of these collectively applying the specifications of the pulsed radar system (Table. I). For the three levels of jamming (Section V.C) the average transmit power calculated was: $63 \mu \mathrm{W}, 630 \mu \mathrm{W}$ and $6.3 \mathrm{~mW}$ respectively; using a jamming antenna with a reasonable gain of $10 \mathrm{dBi}$, a target RCS of $0 \mathrm{dBsm}$ [17] and at a range of $90 \mathrm{~m}$. This was obtained under the following assumptions: the noise temperature, receiver bandwidths and combined losses of the radar and jamming device are equal and that the jamming efficiency $\left(J_{e f f}\right)$ is $100 \%$, meaning the device is jamming over 
the full radar bandwidth. The calculated average power levels in order to generate the desired JSR's are feasible for any of the jamming devices/systems listed in Table. III. This quick analysis delivers an understanding of the roles each radar parameter plays in the context of the experimental setup.

The jamming signals were injected into the range time domain of the radar data, with the methods of implementation described in Table. III The CFAR could operate mostly unaffected, in all cases but the most extreme jamming conditions. In this case the range cells were not able to be isolated therefore the STFT was applied over all range cells to obtain the micro-Doppler signature $(60$ cells $)$. This was necessary as otherwise completely blank spectrograms would be produced, meaning the targeted jamming had done its job and leaving little for the system to respond to. In the context of a real system employing a robust CFAR detector, it may have some further algorithms to be able to cope with various forms of jamming prior to transformations. However, successful classification in later stages would still not be guaranteed due to the distortion present in the resultant spectrograms, as depicted in Fig. 3. Therefore, the rationale behind this implemented penalty is a reasonable compromise between having an operational CFAR and degraded spectrograms.

It should be noted that in the case of targeted pulse compression Fig. 3a, the idea of direct jamming injection does not apply and the method of degradation is not trivial. This is because it is not possible to directly change the modulation of the radar data after the experiments had taken place, the closest equivalent would be to intervene in the match filtering process. This was achieved by altering the frequency response of the reference chirp and defining three worsening levels akin to the potential learning stages taking place in the EA escort vehicle described in Table. III. The first level was defined as a frequency chirp extending over half the true bandwidth of the real chirp signal $(22.5 \mathrm{MHz})$, the second being a $\mathrm{CW}$ signal at $2.4 \mathrm{GHz}$ and the final level being no match filtering process taking place at all; effectively undoing the pulse compression gain of $14.3 \mathrm{~dB}$ as detailed in Section II.
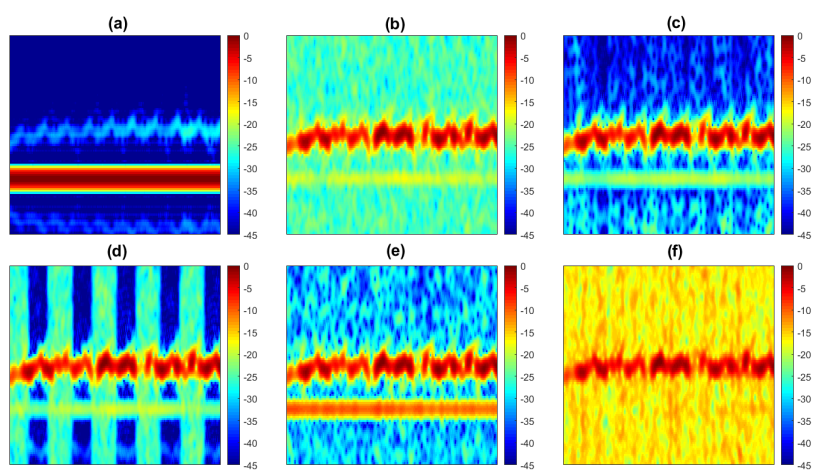

Fig. 3. Collage of spectrograms after synthetic jamming injection into the range time domain; the correlation with the JSR is indicated in brackets: (a) Targeted Pulse Compression (no matched filter); (b) Spot (20 dB);

(c) Cover (random modulations of amplitude proprotional to the noise level,

$20 \mathrm{~dB}$ ); (d) Blinking (20 dB); (e) Unintentional (number of corrupted pulses proportional to noise level, $20 \mathrm{~dB}$ ); (f) Deceptive (number and degree of shuffled pulses across range cells proportional to the noise level $20 \mathrm{~dB}$ )

\section{NETWORK CONFIGURATIONS}

The deep learning architecture chosen for implementation in this work is based upon the popular pre-trained model known as AlexNet [23]. This was chosen as it significantly outperforms other models, such as GoogleNet, Resnet50, Resnet101 and InceptionResnetV2; in a multitude of aspects such as convergence time, network simplicity and training time (for this scenario). DNNs were trained at each radar node meaning that a total of 2800 samples were available and from this, the samples were split into $60 \%$ training, $10 \%$ validation and $30 \%$ test pools. The training data was augmented in real time and consisted of mirroring the spectrograms and CVDs in the $\mathrm{X}$ and $\mathrm{Y}$ planes, any other combination of rotations, sheering, scaling and translations only hindered the obtained classification accuracy. In the context of this work, tuning the specific architecture of the deep learning model was not of importance as it would have led to significantly longer training times, accepting this enabled creativity regarding combinations of these networks, applicable to implementation in multistatic radar systems.

\section{A. Voting System}

The simplest network architecture depicted in the red dashed box in Fig. 4, consists of three independently trained DNNs $\left(n_{x}\right)$, input image pools are represented by $\left(i_{\mathrm{x}}\right)$ and are delivered concurrently to the respective networks. As each network has an output of 14 classes and the CVD is a direct transformation of the spectrogram, this means that they effectively correspond to the same class. Therefore, at each network an internal vote is made between the two transformations as to which one is the most confident in their decision. The intermediate prediction is composed of the most confident prediction of each network for each input test image. Finally, the predictions from the three nodes are then compared and a similar procedure takes place, whereby the final decision is dominated by the most confident radar node.

\section{B. Feed Forward Network}

A more advanced method of combining the decisions from each of the three radar nodes was determined, whereby a feed forward network $\left(F_{n}\right)$ consisting of 21 hidden units was placed between the probabilistic output of each of the three DNNs and funneled back to the original classes and hence the final decision $\left(D_{0}\right)$. Each of the DNNs have 14 outputs, using the same voting process as above this is compressed down to 7 and then aggregated to form a 21-class output. This is then input to the feed forward network (FFN) which is trained on the unused $10 \%$ validation data pool, from this the appropriate internal weights $\left(w_{m, n}\right)$ are determined. The FFN configuration is portrayed by the blue dashed box in Fig. 4.

\section{Feed Forward with Jamming Detection}

The final network configuration consists of an amalgamation of the two previous designs, but in addition to this there is a further network $\left(J_{\Omega}\right)$ running in parallel, which can detect the presence of jamming in any of the three radar nodes. As $J_{\Omega}$ is based on a DNN architecture, this enables it to detect and distinguish between a wide variety of the jamming measures, it also has the ability to discriminate between the three levels. As the synthetic jamming was injected directly 
into the range time data for each of the 18 jamming classes (chosen randomly for each discrete jamming level), the training pool consisted of 191,520 images $(19 \times 2 \times 8400 \times 60 \%)$. This included the spectrogram and CVD versions which lead to total of 38 classes and includes the baseline transformations which have no jamming applied ( 2 classes). To test the system, a Look Up Table (LUT) of permutations was devised consisting of 64 unique combinations $\left(4^{3}\right)$, which is based on the 3 jamming levels plus one uncorrupted (4), to the power of the number of radar nodes (3), this can be seen in Fig. 7.

For each of the permutations a unique FFN was trained on the equivalent jammed images based on the reserved $10 \%$ validation data. This was repeated over 5000 iterations taking the network achieving the highest classification accuracy using the $30 \%$ testing data. With this bank of 64 FFNs, the jamming detector is able to analyze the input image and determine the class and hence the level of jamming. From this it can then switch in the appropriate FFN such that the particular jammed node is weighted appropriately as to retain confidence in the final classification decision $\left(D_{0}\right)$.

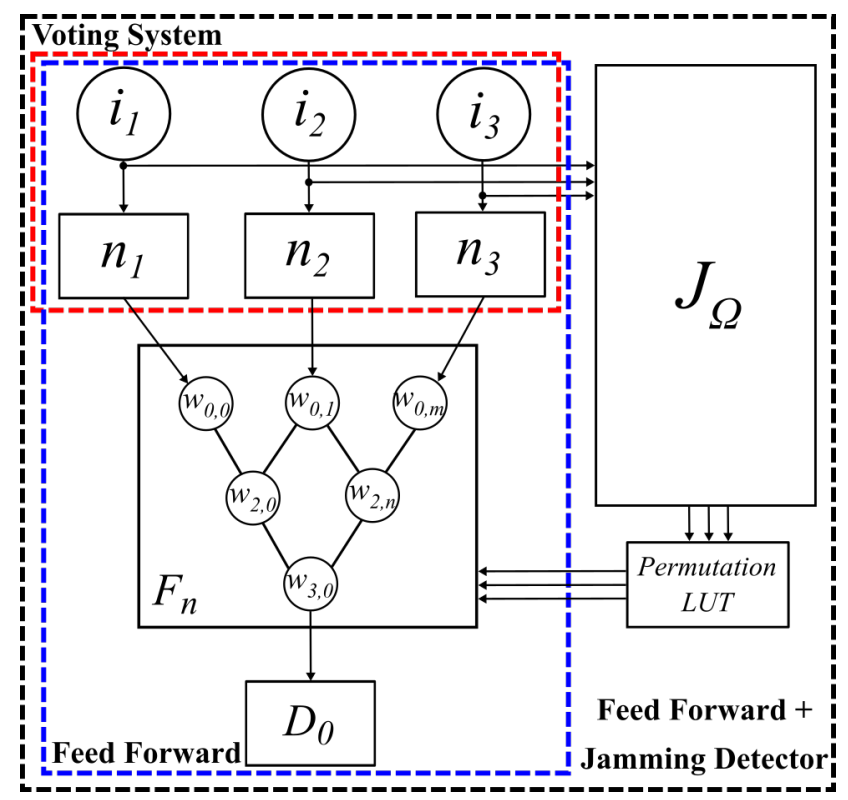

Fig. 4. Diagram of the three progressive network configurations

\section{RESULTS}

Three DNNs based on the AlexNet architecture were trained on a GPU workstation consisting of two Nvidia GTX 1080TI's. The learning rate was set to $1 \mathrm{e}-4$, the mini-batch size to 16 and the maximum epochs to 45 , this led to a training time of three hours per network. Two types of test scenarios have been experimented with in this work: the first compares the results of the voting system to the feed forward system, this uses the unseen $30 \%$ unjammed testing data, the second compares the performance of the automatic jamming detection system with that of the voting system, when the jammed testing data across all permutations are input to the system.

\section{A. Voting System}

The classification accuracy achieved using the unjammed test data and the voting system topology is detailed in Table.
IV. The average accuracy gained from the intermediate voting stage over the simple aggregate average was $6.2 \%$. Finally, an average of $14.3 \%$ is gained utilizing the final voting stage.

TABLE. IV

\begin{tabular}{cccc}
\multicolumn{4}{c}{ VOTING SYSTEM CLASSIFICATION ACCURACY } \\
\hline \hline Scenario / \% & Node 1 & Node 2 & Node 3 \\
\hline Average & 73.3 & 75.2 & 68.9 \\
Spectrogram & 78.1 & 79.1 & 75.6 \\
CVD & 68.5 & 71.3 & 61.8 \\
Internal Vote & 79.2 & 80.7 & 76.1 \\
\hline Final Vote & & 86.8 &
\end{tabular}

The confusion matrix for the final vote is shown in Fig. 5, with the class accuracy having a standard deviation of 8.42 . The key for the classes can be found in Table. II.

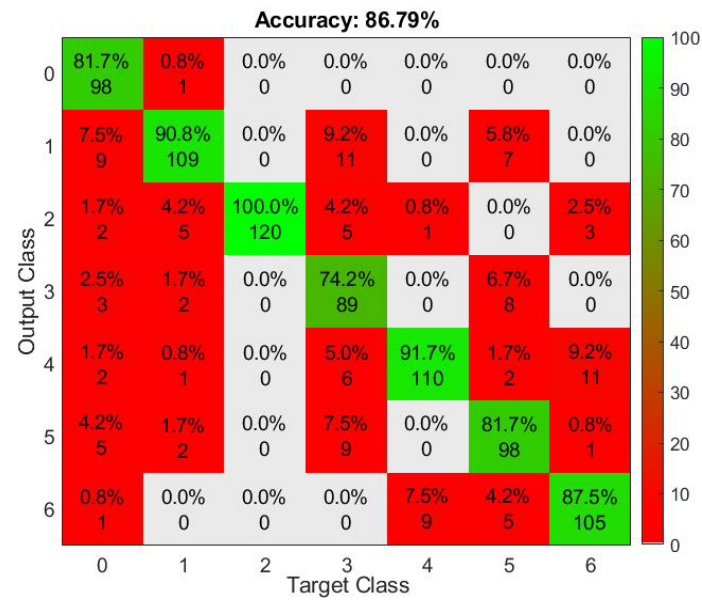

Fig. 5. Confusion matrix for voting system

\section{B. Feed Forward Network}

The FFN obtains the same intermediate classification accuracies as in Table. IV, except for the final decision, where $90.7 \%$ is obtained. The confusion matrix for this test is shown in Fig. 6 and an improvement of 3.9\% is demonstrated over the voting system methodology. The accuracy across the classes appear to be more stable consistently staying over $85 \%$ and this is confirmed through the standard deviation of 4.49 .

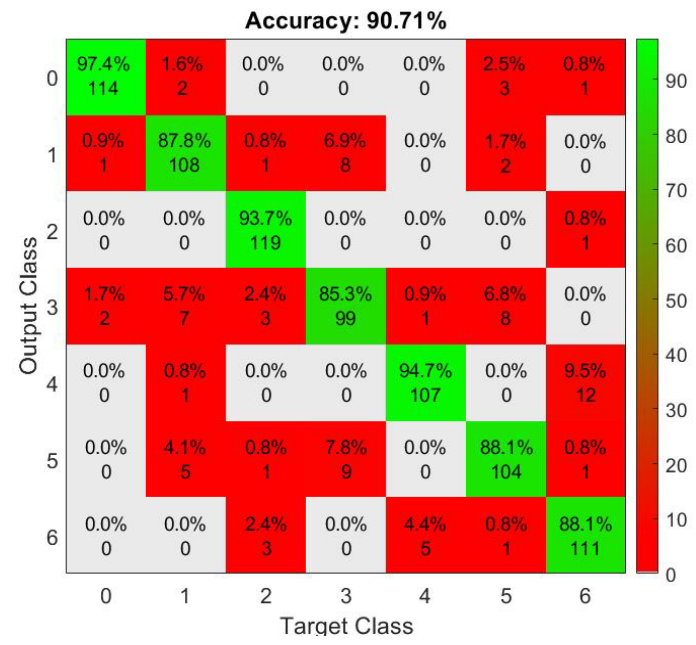

Fig. 6. Confusion matrix for feed forward (FFN) system 


\section{Feed Forward Network with Jamming Detection}

Fig. 7 shows the classification accuracy of the trained FFN bank when the equivalent jammed images from the testing image set are injected into the network. It is important to note that no jamming detection occurs as they have been injected systematically into the correct jamming permutations.



Fig. 7. Classification accuracy of the 64-FFN bank with Jamming Detection

Finally, the jamming detection network $\left(J_{\Omega}\right)$ was trained over a period of five days and was manually stopped when the validation accuracy saturated at $86 \%$. This was more than acceptable considering the large volume of training images used. Following this, the 38 classes consisting of the spectrogram and the CVD which achieved individual accuracies of $89.1 \%$ and $83.1 \%$ respectively, were compressed back down to the original 19 classes, this delivered a performance gain just short of $10 \%$, now yielding a final jamming classification accuracy of $95.5 \%$.

As mentioned in section III.B, three levels of jamming severity were chosen; the SNR on average of each spectrogram was $37 \mathrm{~dB}$ and the third jamming level was chosen to be $30 \mathrm{~dB}$ as this caused the classification accuracy to consistently fall below $20 \%$ in every case (approaching the random guess accuracy of $14.3 \%$ ). The other two jamming levels were chosen to be $10 \mathrm{~dB}$ and $20 \mathrm{~dB}$ respectively, which produced quite an even spread of jamming levels. This resulted in a residual Signal to Jamming Ratio (SJR) of approximately, $7 \mathrm{~dB}, 17 \mathrm{~dB}$ and $27 \mathrm{~dB}$ over the entire dataset.

The classification accuracy of the jamming detector is shown in Fig. 8, this indicates how well the detector is able to match the predicted jamming state to the correct jamming permutation for all three radar nodes. The first 16 permutations exhibit a significant dip of approximately $20 \%$, as the jamming detector only achieves an accuracy of $89 \%$ for cases of nil jamming. This is made worse by the fact that there is only one class for this case as opposed to six for each other forms of jamming. It should also be noted that even if the incorrect FFN is called from the reference bank, outright classification failure is not guaranteed, therefore Fig. 7 and Fig. 8 do not simply multiply to produce Fig. 9.

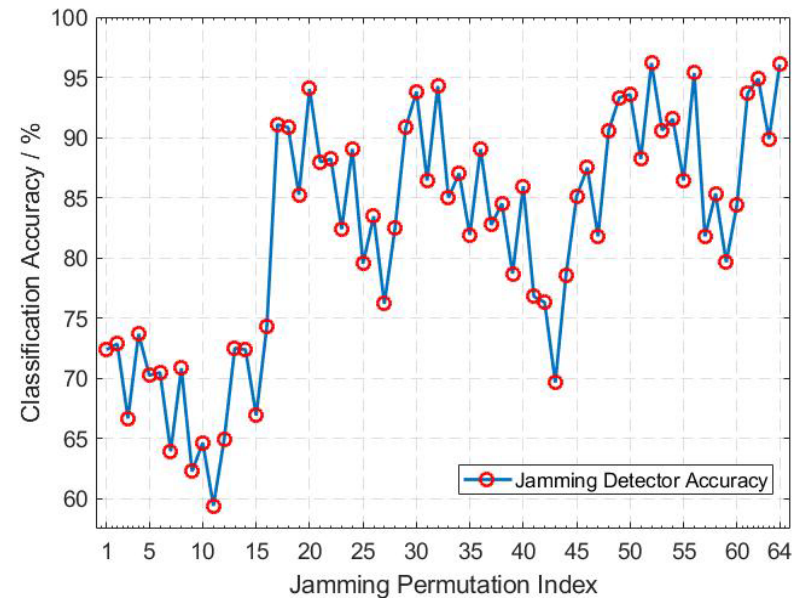

Fig. 8. Accuracy of the jamming detector $\left(J_{\Omega}\right)$ over the permutation indices

Fig. 9, depicts the classification accuracy of the complete system, when the jamming detector is running in parallel with the base DNNs at each node and automatically determining the jamming severity for each successive input image. Following this, it then matches it to the believed permutation index and hence the appropriate network from the 64-FNN reference bank. The red trace shows the accuracy obtained from using the aforementioned complete system from Fig. 4; a mean gain of $16.2 \%$ (green trace) is observed over the voting system (represented by the blue trace). The benefits of the system become evident when the input jamming combinations are particularly severe (50 to 64 ), however there is only so much that the system can cope with in the presence of serious directed jamming into all three of the radar nodes.

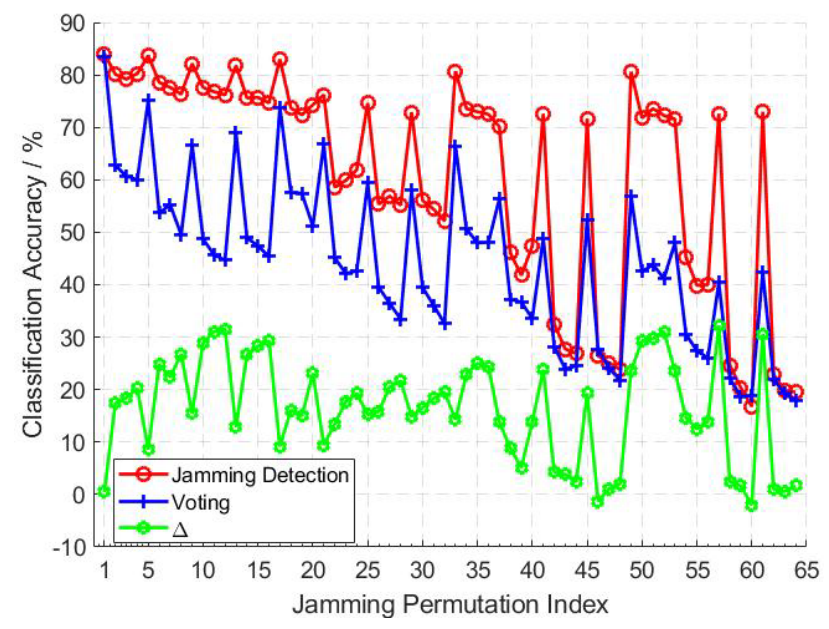

Fig. 9. Accuracy of the jamming system compared with the voting system

The result of using the predictions from only the monostatic radar node is shown in Fig. 10, the four steps observed are due to the discrete jamming levels and the permutation order used for node 1 . Though the comparison of every point is strictly not possible as the red trace encompasses permutations across all three radar nodes, it is still clear that the multistatic detection system offers performance gain, as the permutations within each step only mean that the other two nodes are being jammed to some further degree. The benefits of a multistatic system are especially obvious at the permutation boundaries (indices: 
1, 16, 32 and 48), where an improvement of approximately $4 \%, 25 \%, 49 \%$ and $63 \%$ is observed respectively.

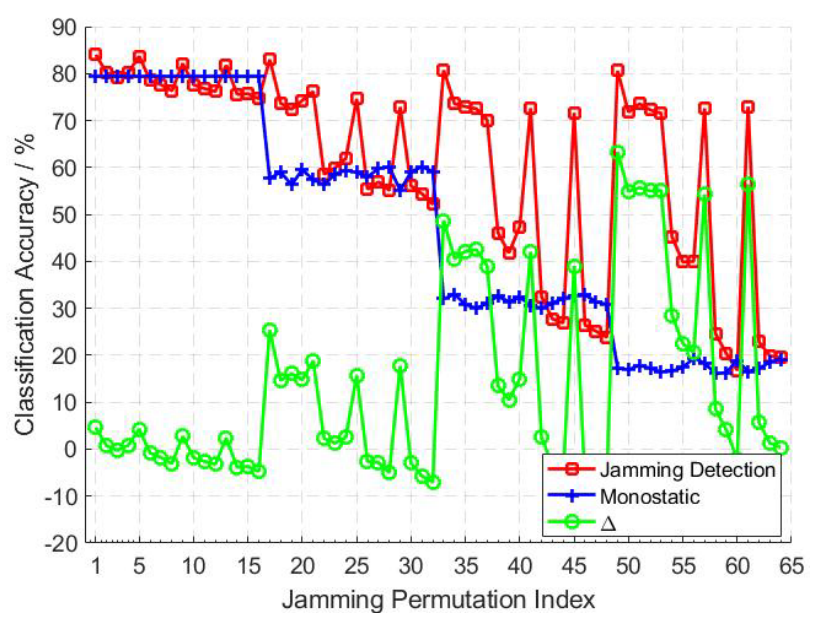

Fig. 10. Classification accuracy of the complete jamming detection system compared with the monostatic setup (node 1)

\section{CONCLUSION}

In this work, real experimental data was gathered from four people performing seven different activities in the field. This data was batch-analyzed to produce two transformations of the same activity (the spectrogram and CVD), this representation diversity has proved to be highly advantageous, raising the accuracy of the jamming classifier $\left(J_{\Omega}\right)$ by $10 \%$. In addition to this, a further data set was generated of the equivalent jammed images of these two transformations in order to train $J_{\Omega}$. A novel classification architecture was then proposed; which consisted of multiple DNNs assigned at each radar node, culminated by a suitable FFN, which is intelligently chosen by the over watch jamming detector $\left(J_{\Omega}\right)$. The performance of this topology was compared against a voting system baseline, which in some respects could be considered the simplest way of fusing the data between the three radar sensors.

The results obtained demonstrate that utilization of FFNs in this sensor system is indeed advantageous even in the simplest of cases, however this architecture begins to really prove itself in the face of targeted jamming; keeping classification accuracy above $70 \%$ for roughly $60 \%$ of the permutations, as opposed to the spurious nature of the voting system. Though, it should be noted that the voting system still has some jamming mitigation capabilities, as the injected jamming may cause the DNN to become unconfident in its final probabilistic decision, therefore handing over responsibility to the other nodes in an attempt to try and salvage a correct decision; this in some cases still functions to a respectable degree.

A wide range of options and parameters were explored in order to improve the classification accuracy of the collective DNNs. The pre-trained DNN AlexNet was found to be superior in terms of classification accuracy, complexity and training time; in addition to this, only X-Y reflections were found to be beneficial when augmenting the training data, which is logical, as the transformations have strong physical kinematic meaning, changing over time. Differing dwell times of $(0.5,1$ and 2.5 seconds) were also briefly investigated and a dwell time of 1 second was found to be the most suitable for this classification problem. This represents a challenging tradeoff between generating a sufficiently sized data set for the DNN and also that each image contains enough visual cues for classification to occur. A further frequency transformation called the cepstrogram [24], which emphasizes periodic details in the spectrogram [19], was also experimented with. This was found to not contain enough information as to warrant its implementation as a useful transformation in the scope of this classification problem.

The notion that multistatic radar provides improved performance over a monostatic system, in the presence of targeted enemy jamming, is thoroughly put through its paces. This is proven through a range of tests and experimentations; but specifically, in Fig. 10, where even just the opportunity of having uncorrupted data is enough to maintain classification accuracy at $80 \%$, as opposed to just $17 \%$ for the monostatic node (in the worst cases of jamming). In addition to this, the multistatic setup can also provide small performance gains of a couple percent due to having three times the amount of information available to the overall system. This would be exaggerated further if the target was in an unfavorable position in the field, but within the coverage of the multistatic network.

This complete classification system architecture as discussed in IV.C, is capable of highly efficient data throughput between sensors, as only probabilistic confidence vectors needs to be shared. Although all three input images are input to the single jamming detection classifier, this DNN could easily be duplicated at each node sharing only the degree to which the node thinks it is being jammed between each node. The system could then make a decision about which FFN to switch in at a centralized node, then producing a final decision as to the class of the target in question. The modular nature of this architecture is particularly advantageous and relaxes demands on data throughput between radar nodes, which is a principle concern and can very easily become an issue over long distances.

\section{FUTURE WORK}

As this is a preliminary investigation into jamming resilient systems using deep learning, there is a large scope for future work to be undertaken. The most distinguished outcome in any classification driven problem, is to obtain higher accuracies. This could potentially be achieved in a multitude of ways: to design and train a custom DNN architecture, enabling the weights for each layer to be determined from scratch, rather than predefined matrices as it is using AlexNet. The training time will be significantly longer, but the advantages to be gained may be favorable. Impressive results were observed in the case of the jamming detector, where the training sets were significantly larger and it was trained over a long period. An investigation into the generation of synthetic data sets may also be of interest, such as the use of Generative Adversarial Networks (GANs), where their use has already been proven in the context of human micro-Doppler [25].

Other methods of sensor fusion could also be considered through careful design [26], or the operation of the current 
architecture could be improved, by giving the jamming detector direct control over the weights rather than having a reference bank. This would require mapping the weight space within the hidden layers mathematically and thorough tests to determine the optimum assignment function for the weights at each jamming level. It should be noted that this would only be worthwhile if there is a need to granulize the jamming levels significantly, this is a metric open for investigation.

This work dealt with the effects caused by known jamming techniques and trained the DNN in a supervised learning fashion. In a practical environment it is not entirely feasible to predict every possible effect caused by targeted jamming, therefore the system may not be able respond in an ideal manner. A thorough examination into this issue is required and will be considered for future work, where the scenario will be treated as an unsupervised learning problem.

Finally, it may also be possible to leverage the properties of machine learning in the form of stacked auto encoders (SAEs) [27], to potentially undo or to actively recover the effects of jamming. This is expected to have a similar effect as the topology described in this work, in that it will be effective up until a specific threshold. A direct expansion to this work would be to have a combination of the topologies, whereby the jamming detector would be able to call upon specific SAE recovery cells to restore as much of the original data as possible, prior to classification.

\section{ACKNOWLEDGMENTS}

The authors would like to thank S. Gürbüz for participating in the field experiments and A. Pingarron, R. Palamà, A. Amiri and C. Horne for assisting during the preparation of the field trial. This work was funded by the IET AF Harvey Prize awarded to H. Griffiths (2013), the UK EPSRC, grant EP/R041679/1 which funded F. Fioranelli and Leonardo Airborne and Space Systems which is sponsoring J. S. Patel.

\section{REFERENCES}

[1] Y. Kim, S. Choudhury, and H. J. Kong, "Application of Micro-Doppler Signatures for Estimation of Total Energy Expenditure in Humans for Walking/Running Activities," IEEE Access, vol. 4, pp. 1548-1557, 2016.

[2] D. Blacknell \& H. Griffiths, Radar Automatic Target Recognition (ATR) and Non-Cooperative Target Recognition (NCTR), IET 2013.

[3] M. Ritchie, F. Fioranelli, H. Borrion, and H. Griffiths, "Multistatic micro-Doppler radar feature extraction for classification of unloaded/loaded micro-drones", IET Radar, Sonar Navig., vol. 11, no. 1, pp. 116-124, 2017.

[4] M. Ritchie, F. Fioranelli, H. Griffiths, and B. Torvik, "Monostatic and bistatic radar measurements of birds and micro-drone," 2016 IEEE Radar Conf. RadarConf 2016, 2016.

[5] F. Fioranelli, M. Ritchie, and H. Griffiths, "Multistatic Human MicroDoppler Classification of Armed / Unarmed Personnel" IET Radar, Sonar Navig., vol. 9, no. 7, pp. 857-865, 2015.

[6] B. Marr., "The Top 10 AI And Machine Learning Use Cases Everyone Should Know About" Forbes 2016, Available [online]: https://www.forbes.com/sites/bernardmarr/2016/09/30/what-are-the-top10 -use-cases-for-machine-learning-and-ai/\#7f2d07ca94c9

[7] F. Fioranelli, M. Ritchie and H. Griffiths, "Aspect angle dependence and multistatic data fusion for micro-Doppler classification of armed/unarmed personnel," in IET Radar, Sonar \& Navigation, vol. 9, no. 9, pp. 1231-1239, 12 2015. doi: 10.1049/iet-rsn.2015.0058
[8] R. Du, Y. Fan and J. Wang, "Pedestrian and Bicyclist Identification Through Micro Doppler Signature With Different Approaching Aspect Angles," in IEEE Sensors Journal, vol. 18, no. 9, pp. 3827-3835, 1 May1, 2018. doi: 10.1109/JSEN.2018.2816594

[9] Li, H., Shrestha, A., Heidari, H., Le Kernec, J., Fioranelli, F.; 'Magnetic and radar sensing for multimodal remote health monitoring', accepted in IEEE Sensors Journal, Sept. 2018.

[10] S. Ryu, J. Suh, S. Baek, S. Hong and J. Kim, "Feature-Based Hand Gesture Recognition Using an FMCW Radar and its Temporal Feature Analysis," in IEEE Sensors Journal, vol. 18, no. 18, pp. 7593-7602, 15 Sept.15, 2018. doi: 10.1109/JSEN.2018.2859815

[11] Y. Lin, J. Le Kernec, S. Yang, F. Fioranelli, O. Romain, and Z. Zhao, "Human Activity Classification with radar: Optimization and Noise Robustness with Iterative Convolutional Neural Networks followed with Random Forests," IEEE Sensors Journal, 2018.

[12] S. Skaria, A. Al-Hourani, M. Lech and R. J. Evans, "Hand-Gesture Recognition Using Two-Antenna Doppler Radar with Deep Convolutional Neural Networks," in IEEE Sensors Journal. doi: 10.1109/JSEN.2019.2892073

[13] G. Klarenbeek, R. I. A. Harmanny and L. Cifola, "Multi-target human gait classification using LSTM recurrent neural networks applied to micro-Doppler," 2017 European Radar Conference (EURAD), Nuremberg, 2017, pp. 167-170. doi: 10.23919/EURAD.2017.8249173

[14] M. S. Seyfioglu, B. Erol, S. Z. Gurbuz and M. G. Amin, "Diversified radar micro-Doppler simulations as training data for deep residual neural networks," 2018 IEEE Radar Conference (RadarConf18), Oklahoma City, OK, 2018, pp. 0612-0617. doi: 10.1109/RADAR.2018.8378629

[15] Y. Li, Z. Peng, R. Pal and C. Li, "Potential Active Shooter Detection Based on Radar Micro-Doppler and Range-Doppler Analysis Using Artificial Neural Network," in IEEE Sensors Journal, vol. 19, no. 3, pp. 1052-1063, 1 Feb.1, 2019. doi: 10.1109/JSEN.2018.2879223

[16] J. S. Patel., F. Fioranelli, M. Ritchie, and H. Griffiths, "Multistatic Radar Classification of Armed Vs Unarmed Personnel Using Neural Networks," Evol. Syst.,vol 9, pp 135-144, Nov. 2017.

[17] G. W. Stimson, H. Griffiths, C. Baker, D. Adamy, 'Stimson's Introduction to Airborne Radar', 3rd Edition, The Institution of Engineering and Technology, 2014

[18] F. Fioranelli, J. Patel, S. Z. Gurbuz, M. Ritchie and H. Griffiths "Multistatic human micro-Doppler classification with degraded/jammed radar data", Accepted in IEEE RadarConf, Boston 2019.

[19] J. S. Patel., C. Al-Ameri, F. Fioranelli, D. Anderson, "Multi-Time Frequency Analysis \& Classification of a Micro Drone Carrying Payloads Using Multistatic Radar" Accepted in IET Radar Conf 2018.

[20] T. E. Derham, S. Doughty, K. Woodbridge, C. J. Baker, 'Design and evaluation of a low-cost multistatic netted radar system', IET Radar, Sonar \& Navigation, vol. 1, pp. 362-368, 2007.

[21] Miller A. W., Clemente C., Robinson A., Greig D., Kinghorn A. M., and Soraghan J. J., "Micro-Doppler based target classification using multifeature integration", IET Intelligent Signal Processing Conf. (ISP) 2013 pp. 1-6.

[22] D. Adamy, 'A First Course in Electronic Warfare EW101', $1^{\text {st }}$ Edition, Artech House, 2001.

[23] A. Krizhevsky, I. Sutskever, and G. E. Hinton, "ImageNet Classification with Deep Convolutional Neural Networks," Adv. Neural Inf. Process. Syst., pp. 1-9, 2012.

[24] B. P. Bogert, M. J. R. Healy and J. W Tukey. (1963): “The Quefrency Analysis of Time Series for Echoes: Cepstrum, Pseudo Autocovariance, Cross-Cepstrum and Saphe Cracking", in: Proc. Of Time Series Analysis, Wiley, NY, Chapter 15, p. 209-243.

[25] X. Shi, Y. Li, F. Zhou and L. Liu, "Human Activity Recognition Based on Deep Learning Method," 2018 International Conference on Radar (RADAR), Brisbane, QLD, 2018, pp. 1-5. doi: 10.1109/RADAR.2018 .8557335

[26] J. R. Raol, 'Multi-Sensor Data Fusion with MATLAB', $1^{\text {st }}$ Edition, CRC Press, Taylor Francis

[27] B. Wang, L. Du, Y. Ma and H. Liu, "A Bayesian denoising mehod for complex radar signal with application to classification of human individuals," 2015 IEEE China Summit and International Conference on Signal and Information Processing (ChinaSIP), Chengdu, 2015, pp. 268272. doi: 10.1109/ChinaSIP.2015.723040 